\title{
On Martingale Transformations of Multidimensional Brownian Motion
}

\author{
M. Mania ${ }^{1)}$ and R. Tevzadze ${ }^{2)}$
}

1) A. Razmadze Mathematical Institute of Tbilisi State University and Georgian-American University, Tbilisi, Georgia,

(e-mail: misha.mania@gmail.com)

2) Georgian-American University and Institute of Cybernetics of Georgian Technical Univercity, Tbilisi, Georgia

(e-mail: rtevzadze@gmail.com)

\begin{abstract}
We describe the class of functions $f: R^{n} \rightarrow R^{m}$ which transform a vector Brownian Motion into a martingale and use this description to give martingale characterization of the general measurable solution of the multidimensional Cauchy functional equation.
\end{abstract}

2010 Mathematics Subject Classification. 60G44, 60J65, 97 I70

Keywords: Brownian Motion, Martingales, Functional Equations.

\section{Introduction}

It is well known (see, e.g., [18], [5], [4], [12]) that if $f=(f(x), x \in R)$ is a function of one variable and $W$ is linear Brownian Motion then the transformed process $f\left(W_{t}\right)$ is a continuous (or right-continuous) martingale if and only if $f$ is an affine function. In multidimensional case this result is no longer true. A simple countre-example gives the non-linear function $f(x, y)=$ $x^{2}-y^{2}$ for which the transformed process $f\left(W_{t}^{1}, W_{t}^{2}\right)$ of two independent Brownian Motions $W^{1}$ and $W^{2}$ is a continuous martingale. Our goal is to 
give sufficient (and necessary) conditions in multidimensional case, when martingale function is affine or almost affine, i.e., it coincides with an affine function almost everywhere with respect to the Lebesgue measure.

Let $W=\left(W_{t}, t \geq 0\right)$ be a $n$-dimensional standard Brownian Motion defined on a probability space $(\Omega, \mathcal{F}, \mathcal{P})$ with filtration $F=\left(\mathcal{F}_{t}, t \geq 0\right)$ satisfying the usual conditions of right-continuity and completeness.

In [2] the functions $f: R^{n} \rightarrow R^{m}$ was studied such that $f(W)$ is Brownian path preserving, i.e., is a standard Brownian Motion up to a random time change. It follows from their results that, if $m=1$ and $f$ is continuous, then the process $f(W)$ is Brownian path preserving if and only if $f$ is harmonic. It was shown in [11] that if $f(W)$ is again a Brownian motion (without allowing time change) with respect to the same filtration, then $f$ is an affine function. In Theorem 1 we consider the case when the transformed process $f(W)$ is a general martingale, without assuming the continuity of paths, but impose an additional condition, which is satisfied in the case of Brownian motion and which guaranties the almost linearity of the function $f$ and the linearity if we additionally assume the continuity of $f$.

In section 3 we apply these results to give an equivalent martingale characterization of the general measurable solution of multidimensional Cauchy's functional equation (see, e. g., [1], [15] and [10, 6] for almost additive version). In Theorem 3 we show that if $f=\left(f(x), x \in R^{n}\right)$ is a measurable function satisfying the Cauchy functional equation

$$
f(x+y)=f(x)+f(y),
$$

for almost all $(x, y)$ in the sense of the Lebesgue measure on $R^{2 n}$, then the transformed process $f\left(W_{t}\right)$ is a martingale which satisfies condition $\mathrm{C}$ ) of Theorem 1 and, hence is almost affine function.

\section{Martingale functions of Brownian Motion}

Let $M$ be a martingale with respect to the filtration $F_{\tilde{N}}^{W}=\left(\mathcal{F}_{t}^{W}, t \geq 0\right)$ generated by the Brownian Motion $W$ and denote by $\tilde{M}$ the continuous modification of $M$. Since almost all paths of $\tilde{M}$ are continuous, $\tilde{M}$ is locally square integrable martingale and the square characteristic $\langle\tilde{M}\rangle$ of $\tilde{M}$ exists. We shall call $\langle\tilde{M}\rangle$ the square characteristic of $M$ also, i.e., $\langle M\rangle \equiv\langle\tilde{M}\rangle$.

Theorem 1. Let $f(x)=\left(f_{1}(x), \ldots, f_{m}(x)\right), x \in R^{n}$ be a measurable function, such that $f\left(W_{t}\right)=\left(f_{1}\left(W_{t}\right), \ldots, f_{m}\left(W_{t}\right)\right)$ is a martingale satisfying 
condition:

$C)$ the process $\left\langle f_{j}(W)\right\rangle_{t}-C_{j} t$ is a non-decreasing for some $C_{j}, j=1, \ldots, n$.

Then $f(x)$ must be an affine function for almost all $x$ in the sense of the Lebesgue measure on $R^{n}$.

Proof. Let

$$
g(t, x)=E\left(f\left(W_{T}\right) \mid W_{t}=x\right) .
$$

It is evident that $g(t, x)$ satisfies the generalized heat equation

$$
\int_{0}^{T} \int_{R^{n}} g(s, y)\left(\frac{\partial \varphi}{\partial t}(s, y)-\frac{1}{2} \Delta \varphi(s, y)\right) d y d s=0
$$

for every infinitely differentiable finite $\left(\right.$ on $\left.[0, T] \times R^{n}\right)$ function $\varphi$.

By the Markov property of the Brownian motion

$$
g\left(t, W_{t}\right)=E\left(f\left(W_{T}\right) \mid \mathcal{F}_{t}\right) \quad \text { a.s. }
$$

and from the martingale property of $f\left(W_{t}\right)$ we have that for all $t \leq T$

$$
g\left(t, W_{t}\right)=f\left(W_{t}\right) \quad \text { a.s. }
$$

Therefore, for all $t \leq T$

$$
\int_{R^{n}}|g(t, x)-f(x)| \frac{1}{\sqrt{2 \pi t}} e^{-\frac{|x|^{2}}{2 t}} d x=0
$$

which implies that for all $t \leq T$

$$
g(t, x)=f(x) \quad \text { a.e }
$$

with respect to the Lebesgue measure.

Therefore, it follows from (11) that

$$
\int_{0}^{T} \int_{R^{n}} f(y)\left(\frac{\partial \varphi}{\partial t}(s, y)-\frac{1}{2} \Delta \varphi(s, y)\right) d y d s=0
$$


for every infinitely differentiable finite (on $\left.[0, T] \times R^{n}\right)$ function $\varphi$.

Taking $\varphi(t, y)=\varphi_{0}(t) \varphi_{1}(y)$ with $\int_{0}^{T} \varphi_{0}(t) d t=1$ we obtain from (2) that

$$
\int_{R^{n}} f(y) \Delta \varphi_{1}(y) d y=0
$$

for every infinitely differentiable finite on $R^{n}$ function $\varphi_{1}$. From Theorem of [17] follows that there exists infinite differentiable (even analytic) harmonic function $\tilde{f}$ such that $f(x)=\tilde{f}(x)$-a.e. . It is clear that $\tilde{f}\left(W_{t}\right)$ is continuous modification of $f\left(W_{t}\right)$. By the Ito formula we get

$$
\tilde{f}_{j}\left(W_{t}\right)=\tilde{f}(0)+\int_{0}^{t} \nabla \tilde{f}_{j}\left(W_{s}\right) d W_{s}
$$

which means $\left\langle\tilde{f}_{j}(W)\right\rangle_{t}=\int_{0}^{t} \tilde{f}_{j}\left(W_{s}\right)^{2} d s$. The condition of Theorem gives $\left|\nabla \tilde{f}_{j}\left(W_{s}\right)\right|^{2} \leq C_{j}^{2}$ a.e. which is the same as $\left|\nabla \tilde{f}_{j}(x)\right| \leq C_{j}$. By the mean value theorem we obtain

$$
\left|\tilde{f}_{j}(x)\right| \leq\left|\tilde{f}_{j}(0)\right|+C_{j}|x|
$$

Therefore, the Liouville Theorem ([17], 290p.) implies that among harmonic functions in the whole space only affine functions satisfy this condition.

Corollary 1. Let conditions of Theorem 1 are satisfied. If in addition $f$ is continuous, then it coincides with an affine function.

Sometimes another kind of condition will be useful. Let us introduce the notation

$$
W_{t}^{i j}=\left(W_{1}(t), \ldots, W_{i-1}(t), \frac{W_{i}(t)+W_{j}(t)}{\sqrt{2}}, W_{i+1}(t), \ldots, W_{n}(t)\right) .
$$

Theorem 2. Let $f(x)=\left(f_{1}(x), \ldots, f_{m}(x)\right), x \in R^{n}$ be a measurable function, such that the processes $\left(f\left(W_{t}^{i j}\right), t \geq 0\right)$ are martingales for each $i \leq j$. Then $f(x)$ is an affine function for almost all $x$ in the sense of the Lebesgue measure on $R^{n}$.

Proof. As in Theorem 1 we can prove that $f$ a.e. coincides with an analytic function $\tilde{f}$ satisfying $\Delta \tilde{f}(x)+\sqrt{2}^{1-\delta_{i j}} \frac{\partial^{2} \tilde{f}}{\partial x_{i} \partial x_{j}}(x)=01$ for each $i, j$. If

\footnotetext{
${ }^{1} \delta_{i j}$ denotes Kroneker's delta
} 
we take $i=j$ and subtract equations for different $i$ and $j$ we get $\frac{\partial^{2} \tilde{f}}{\partial x_{i}{ }^{2}}(x)=$ $\frac{\partial^{2} \tilde{f}}{\partial x_{j}{ }^{2}}(x)$. Hence

$$
\frac{\partial^{2} \tilde{f}}{\partial x_{i}{ }^{2}}(x)=\frac{1}{n+1}\left(\Delta \tilde{f}(x)+\frac{\partial^{2} \tilde{f}}{\partial x_{i}{ }^{2}}(x)\right)=0
$$

and

$$
\frac{\partial^{2} \tilde{f}}{\partial x_{i} \partial x_{j}}(x)=\frac{1}{\sqrt{2}} \Delta \tilde{f}(x)+\frac{\partial^{2} \tilde{f}}{\partial x_{i} \partial x_{j}}(x)=0 \text { for } i \neq j .
$$

It follows from these equalities that $\frac{\partial^{2} \tilde{f}}{\partial x_{i} \partial x_{j}}(x)=0$ for any $i, j$, which implies that $\tilde{f}$ is an affine function. Hence, $f$ coincides with an affine function almost everywhere with respect to the Lebesgue measure on $R^{n}$

Corollary 2. Let $f=(f(x), x \in R)$ be a function of one variable and $W$ is a linear Brownian Motion. Then

a) If the process $f\left(W_{t}\right)$ is a martingale, then the function $f$ coincides with an affine function almost everywhere with respect to the Lebesgue measure on $R$.

b) If $f\left(W_{t}\right)$ is a continuous (or right-continuous) martingale then $f$ is an affine function.

Proof. a) follows from Theorem 2. Assertion b) follows from Theorem 2 and the fact that if the process $f\left(W_{t}\right)$ is right-continuous, then the function $f=(f(x), x \in R)$ will be continuous (see Lemma A1 from the Appendix).

\section{An application to functional equations}

In this section we give martingale characterization of the general measurable solution of the multidimensional Cauchy functional Equation.

Theorem 3. For a function $f: R^{n} \rightarrow R^{m}$ the following assertions are equivalent:

i) $f(x)=\left(f_{1}(x), \ldots, f_{m}(x)\right), x \in R^{n}$ is a measurable function satisfying the Cauchy functional equation

$$
f(x+y)=f(x)+f(y),
$$

for almost all $(x, y)$ in the sense of the Lebesgue measure on $R^{2 n}$.

ii) $f(x)=\left(f_{1}(x), \ldots, f_{m}(x)\right), x \in R^{n}$ is a measurable function such that the transformed process $\left(f\left(W_{t}\right), t \geq 0\right)$ is a martingale satisfying condition C (of Theorem 1). 
iii) $f(x)=A x$ for some $m \times n$ constant matrix $A$ for almost all $x$ in the sense of the Lebesgue measure on $R^{n}$.

Proof. $i) \rightarrow i i)$. It is sufficient to show that $f_{1}(W)$ is a martingale satisfying condition $C$ ).

Since for each pair $(\xi, \eta)$ of random vectors with non-degenerate normal distribution (with density function $\rho(x, y)$ )

$P\left(f_{1}(\xi+\eta)-f_{1}(\xi)-f_{1}(\eta) \neq 0\right)=\int_{R^{n}} \int_{R^{n}} I_{\left(f_{1}(x+y)-f_{1}(x)-f_{1}(y) \neq 0\right)} \rho(x, y) d x d y=0$,

we have that

$$
f_{1}(\xi+\eta)=f_{1}(\xi)+f_{1}(\eta) \text { a.s. }
$$

Let $\tilde{\xi}, \tilde{\eta}$ be i.i.d. random vectors with normal distribution. Then, since the pairs $(\tilde{\xi}, \tilde{\eta})$ and $(\tilde{\xi}-\tilde{\eta}, \tilde{\eta})$ have non-degenerate normal distributions, it follows from (5) that

$$
\begin{aligned}
& f_{1}(\tilde{\xi}+\tilde{\eta})=f_{1}(\tilde{\xi})+f_{1}(\tilde{\eta}) \quad \text { a.s. } \\
& f_{1}(\tilde{\xi}-\tilde{\eta})=f_{1}(\tilde{\xi})-f_{1}(\tilde{\eta}) \quad \text { a.s. }
\end{aligned}
$$

To show that $f_{1}(\tilde{\xi})$ is integrable we shall use the idea from [16] on application of the Bernstein theorem.

Let

$$
X=f_{1}(\tilde{\xi}) \text { and } Y=f_{1}(\tilde{\eta}) .
$$

Then from (66) and (7) we have that

$$
\begin{array}{ll}
X+Y=f_{1}(\tilde{\xi}+\tilde{\eta}) & \text { a.s. } \\
X-Y=f_{1}(\tilde{\xi}-\tilde{\eta}) & \text { a.s. }
\end{array}
$$

Since $\tilde{\xi}+\tilde{\eta}$ and $\tilde{\xi}-\tilde{\eta}$ are independent, the random variables $f_{1}(\tilde{\xi}+\tilde{\eta})$ and $f_{1}(\tilde{\xi}-\tilde{\eta})$ will be also independent. Therefore Bernstein's theorem [3] (see also [14]) implies that $X=f_{1}(\tilde{\xi})$ (and $Y=f_{1}(\tilde{\eta})$ ) is normally distributed. Hence the random variable $f_{1}(\tilde{\xi})$ is square integrable. In particular, this implies that

$$
E f_{1}^{2}\left(W_{t}\right)<\infty, \text { for every } t \geq 0 .
$$

Now let us show that $E f_{1}(\tilde{\xi})=0$ for every normally distributed random variable $\tilde{\xi}$ with zero mean. Since $\tilde{\xi}$ and $\tilde{\eta}$ are independent with equal normal distribution we have that the random variables $\tilde{\xi}+\tilde{\eta}, \tilde{\xi}-\tilde{\eta}$ and $\sqrt{2} \tilde{\xi}$ have the same normal distribution, hence

$$
E f_{1}(\tilde{\xi}+\tilde{\eta})=E f_{1}(\tilde{\xi}-\tilde{\eta})=E f_{1}(\sqrt{2} \tilde{\xi}) .
$$


Therefore, from (6) and (17) we obtain that

$$
0=E f_{1}(\tilde{\xi})-E f_{1}(\tilde{\eta})=E f_{1}(\sqrt{2} \xi)=E f_{1}(\tilde{\xi})+E f_{1}(\tilde{\eta})=2 E f_{1}(\tilde{\xi}) .
$$

In particular,

$$
E f_{1}\left(W_{t}-W_{s}\right)=0 \text { for all } s \leq t .
$$

Substituting $\xi=W_{t}-W_{s}$ and $\eta=W_{s}$ in (5) we have that

$$
f_{1}\left(W_{t}\right)-f_{1}\left(W_{s}\right)=f_{1}\left(W_{t}-W_{s}\right) \text { a.s. }
$$

Since $W_{t}-W_{s}$ is independent of $\mathcal{F}_{s}$, taking conditional expectations in this equality from (11) we obtain the martingale equality

$\left.E\left(f_{1}\left(W_{t}\right)-f_{1}\left(W_{s}\right) \mid \mathcal{F}_{s}\right)=E\left(f_{1}\left(W_{t}-W_{s}\right)\right) \mid \mathcal{F}_{s}\right)=E f_{1}\left(W_{t}-W_{s}\right)=0 \quad$ a.s

Denote $C(t)=\operatorname{sign}(t) E f_{1}^{2}\left(W_{|t|}\right), t \in R$. It follows from (5) and the equality $E f_{1}\left(W_{t+s}-W_{t}\right)=0, t, s>0$ that

$$
\begin{array}{r}
C(t+s)=E f_{1}^{2}\left(W_{t+s}-W_{t}+W_{t}\right)=E f_{1}^{2}\left(W_{t+s}-W_{t}\right) \\
+2 E f_{1}\left(W_{t+s}-W_{t}\right) E f_{1}\left(W_{t}\right)+E f_{1}^{2}\left(W_{t}\right)=C(t)+C(s), t, s>0 .
\end{array}
$$

From $C(t)=C(s)+C(t-s), t>s>0$ follows that

$$
\begin{array}{r}
C(t+(-s))=C(t-s)=C(t)-C(s)=C(t)+C(-s), \\
C(-t+s)=-C(t+(-s))=-C(t)-C(-s)=C(-t)+C(s),
\end{array}
$$

which means $C(t+s)=C(t)+C(s)$ holds for all $t, s \in R$. Thus, $C(t)$ is a bounded from below (on $R_{+}$) solution of the Cauchy one dimensional functional equation

$$
C(t+s)=C(t)+C(s), \quad s, t \in R
$$

By well known result [7] the general solution bounded from below on some interval is of the form $C(t)=c t$ for some $c \in R$. Therefore, $E f_{1}^{2}\left(W_{t}\right)=c t$ for some $c>0$. From the equality $\left.E\left(f_{1}\left(W_{t}\right)-f_{1}\left(W_{s}\right)\right)^{2} \mid \mathcal{F}_{s}\right)=E\left(f_{1}\left(W_{t}\right)-\right.$ $\left.f_{1}\left(W_{s}\right)\right)^{2}=c(t-s)$ we obtain that $c t$ is the square characteristic of the continuous modification of the martingale $f_{1}\left(W_{t}\right)$, which means that conditions of Theorem 1 are satisfied. 
$i i) \rightarrow i i i)$. It follows from Theorem 1 follows that $f(x)=A x+B$ for almost all $x$ in the sense of Lebesgue measure on $R^{n}$, for some $m \times n$ matrix $A$ and $B \in R^{m}$. This implies that

$$
f(\xi)=A \xi+B \quad \text { a.s. }
$$

for every normally distributed random variable $\xi$ with zero mean. Since $E f(\xi)=A E \xi=0$, taking mathematical expectations in the last equality we obtain that $B=0$. Thus, $f(x)=A x$ for almost all $x$ in the sense of Lebesgue measure.

iii) $\rightarrow i$ ). If $f(x)=A x$ a.e. for some $m \times n$ constant matrix $A$, for independent random vectors $\xi$ and $\eta$ having the standard normal distribution we have that

$$
P(f(\xi)=A \xi)=P(f(\eta)=A \eta)=P(f(\xi+\eta)=A(\xi+\eta))=1 .
$$

Therefore, avoiding three null sets we obtain from here that $P$ - a.s.

$$
f(\xi+\eta)=f(\xi)+f(\eta)
$$

which implies that (5) is satisfied for almost all $(x, y)$ in the sense of the Lebesgue measure on $R^{2 n}$.

Remark. In one-dimensional case Theorem 3 (more exactly, the equivalence of i) and iii)) follows from [10] and [6], where general additive functions are considered.

The following assertion is well known (see, e.g., [1], [15]). Theorem 3 gives a probabilistic proof of this result.

Corollary 3. Let $f(x)=\left(f_{1}(x), \ldots, f_{m}(x)\right), x \in R^{n}$ be a measurable function satisfying the Cauchy functional equation

$$
f(x+y)=f(x)+f(y),
$$

for all $(x, y) x, y \in R^{n}$. Then $f(x)=A x$ for some $m \times n$ constant matrix $A$.

Proof. It follows from the proof of Theorem 3 that the process $f\left(W_{t}\right)$ is a martingale which satisfies conditions of Theorem 1. From (12) we have that

$$
f\left(x+W_{t}\right)=f(x)+f\left(W_{t}\right),
$$

which implies that the process $f\left(x+W_{t}\right)$ is a martingale for any $x \in R^{n}$. By the martingale equality we have that

$$
f(x)=E f\left(x+W_{t}\right)=\int_{R} f(x+y) \frac{1}{\sqrt{2 \pi t}} e^{-\frac{y^{2}}{2 t}} d y=
$$




$$
=\int_{R} f(y) \frac{1}{\sqrt{2 \pi t}} e^{-\frac{(y-x)^{2}}{2 t}} d y .
$$

Since $E\left|f\left(x+W_{t}\right)\right|<\infty$, equality (13) implies that the function $f(x)$ is continuous and the proof follows from Corollary 1 of Theorem 1.

\section{A Appendix}

Lemma A1. Let $\left(X_{t}, t \geq 0\right)$ be a continuous function such that

$$
X_{0}=0, \limsup _{t \rightarrow \infty} X_{t}=\infty, \liminf _{t \rightarrow \infty} X_{t}=-\infty .
$$

If the composition $Y_{t}=h\left(X_{t}\right)$ is a right-continuous function, then the function $(h(x), x \in R)$ will be continuous.

Proof. It is sufficient to show, that $h$ is continuous on each intervals $[0, b),(-b, 0], b>0$. Let

$$
\begin{array}{r}
\tau(b)=\inf \left\{t>0 ; X_{t} \geq b\right\}, \tau(b, 0)=\inf \left\{t>\tau(b) ; X_{t} \leq 0\right\}, \\
\alpha(x)=\sup \left\{t \leq \tau(b) ; X_{t}=x\right\}, \beta(x)=\sup \left\{\tau(b)<t<\tau(b, 0) ; X_{t}=x\right\}, \\
0 \leq x<b .
\end{array}
$$

It is evident, that $\alpha(x)$ is non-decreasing and right-continuous, and $\beta(x)-$ non-increasing and left-continuous. For example, the right-continuity of $a(x)$ is valid, since if $x_{n} \downarrow x, \alpha\left(x_{n}\right) \downarrow \alpha^{*} \geq \alpha(x)$ then $x_{n}=Y_{\alpha\left(x_{n}\right)} \downarrow Y_{\alpha^{*}}=$ $x, \alpha^{*} \leq \alpha(x)$. Equality $h(x)=Y_{\alpha(x)}=Y_{\beta(x)}$ gives the continuity of $h$ on $[0, b)$. Similarly can be shown the continuity on $(-b, 0]$.

In particular, Lemma A1 implies that, if for a linear Brownian Motion $W$ the process $f\left(W_{t}\right)$ is a.s. right-continuous martingale, then the function $f=(f(x), x \in R)$ is continuous. The following counterexample shows that in multidimensional case this fact is no longer true.

Counterexample. Let $W=\left(W^{1}, W^{2}\right)$ be a two-dimensional Brownian Motion and let $h\left(x_{1}, x_{2}\right)=1_{(1,1)}\left(x_{1}, x_{2}\right)$. Since

$$
P\left(\omega: h\left(W_{t}\right)=0, \forall t \geq 0\right)=P\left(\omega: W_{t} \neq(1,1), \forall t \geq 0\right)=0,
$$

by noting that the filtration $\mathcal{F}_{t}$ is complete, $\left(h\left(W_{t}\right), \mathcal{F}_{t}\right)$ is a martingale (the process indistinguishable from zero), but the function $h$ is not continuous. Note that, $h(x)=0$ almost everywhere with respect to the Lebesgue measure, which is in accordance with Theorem 1. 
Remark. If $h\left(x_{1}, x_{2}\right)=x_{1} I_{\left(R^{2}-(1,1)\right)}\left(x_{1}, x_{2}\right)$ then it follows from (14) that the process $f\left(W_{t}\right)$ is indistinguishable from the Brownian Motion $W_{t}^{1}$, but the function $h$ is not continuous. Therefore, in theorem 2 from [11] or the continuity of $f$ should be required, or the conclusion on almost surely linearity should be made.

In multidimensional case similar to Lemma A1 assertion will be valid, if we require the continuity of the composite function $h\left(X_{t}\right)$ for any continuous function $X_{t}$ (and not only for almost all continuous paths with respect to the Wiener measure).

Lemma A2. Let $h(x), x \in R^{n}$ be real-valued function such that $h\left(X_{t}\right)$ is continuous for each continuous $X_{t}, t \geq 0$. Then $h$ is continuous.

Proof. Let $x_{k}, k=1,2, .$. be a convergent sequence and $x_{0}=\lim _{k \rightarrow \infty} x_{k}$. The function defined by

$$
X_{t}=\left\{\begin{array}{l}
x_{0}, \text { if } t=0 \\
x_{k+1}+k(k+1)\left(t-\frac{1}{k+1}\right)\left(x_{k}-x_{k+1}\right), \text { if } \frac{1}{k+1}<t \leq \frac{1}{k} \\
x_{1}, \text { if } t>1 .
\end{array}\right.
$$

is continuous. Hence $h\left(X_{t}\right)$ is continuous and

$$
\lim _{k \rightarrow \infty} h\left(x_{k}\right)=\lim _{t \rightarrow 0} h\left(X_{t}\right)=h\left(X_{0}\right)=h\left(x_{0}\right) .
$$

\section{References}

[1] J. Aczel and J. Dhombres, Functional equations in several variables, Cambridge Academic Press, 1989.

[2] A. Bernard, E.A. Campbell and A.M. Davie, Brownian motion and generalized analytic and inner functions, Ann. Inst. Fourier 29 (1979), 207228.

[3] S. N. Bernstein, Ob odnom svoystve, harakterizuyushhem zakon Gaussa (in Russian) [On a characteristic property of the normal law]. Tr. leningr.Polytech. Inst.3,21-22 (1941)

[4] R. Chitashvili and M. Mania, On functions transforming Wiener process into semimartingale. Probab. Theory Related Fields, V. 109, 1997, p.5776 . 
[5] E. Cinlar, J. Jacod, P. Protter and M. J. Sharpe, Semimartingales and Markov processes. Z. Warscheinlichkeitstheor. Verw. Geb. V. 54, (1980), pp.161-218.

[6] N. G. De Bruijn, On Almost Additive Functions, Colloquium Mathematicum , Vol.XV, 1966, 59-63.

[7] J. Diedonne, Foundations of Modern Analysis, Academic Press, 1969.

[8] I. Karatzas and S. E. Shreve, Brownian Motion and Stochastic Calculus, Springer, 1991

[9] H. Kestelman, On the functional equation $f(x+y)=f(x)+f(y)$, Fund. Math. 34 (1947), 144-147.

[10] W. Jurkat, On Cauchy's functional equation, Proceedings of the American Mathematical Society, Vol. 16, No. 4, 1965, pp. 683-686.

[11] M. Tehranchi, If $\mathrm{B}$ and $\mathrm{f}(\mathrm{B})$ are Brownian motions, then $\mathrm{f}$ is affine, Rocky Mountain J. Math. Volume 47, Number 3 (2017), pp. 947-953.

[12] M. Mania and R. Tevzadze, Semimartingale functions for a class of diffusion processes. (in Russian) Teor. Veroyatnostei i Primenenija. 45 (2000), No. 2, 374-380; English transl.: Theory Probab. Appl. Vol. 45, No. 2 (2000), pp. 337-343 .

[13] A. Ostrowski, ber die Funktionalalgleichung der Exponentialfunktion und verwandte Funktionalgleichungen. Jber. Deutch. Math. Verein 38 (1929), 54-62.

[14] M. P. Quine, On Three Characterizations of the Normal Distribution, Probability Theory and Mathematical Statistics, Vol 14, Fasc 2, (1993), pp. 257-263.

[15] P. K. Sahoo, P. Kannappan, Introduction to Functional Equation, CRC Press, Boca Raton, 2011.

[16] S. N. Smirnov, A probabilistic note on the Cauchy functional equation, Aequat. Math.Vol. 93, Issue 2, (2019), pp. 445-449.

[17] V. S. Vladimirov, Equations of Mathematical Physics, 1984. 
[18] A. T. Wang, Generalized Itô's formula and additive functionals of Brownian motion, Zeit. für Warscheinlichkeit., V. 41, (1977), p.153-159. 